
éducation relative à l'environnement

\title{
La communauté d'apprentissage en éducation relative à l'environnement : une nouvelle stratégie dans un processus de changements éducationnels
} Isabel Orellana

\section{(2) OpenEdition}

\section{Journals}

Édition électronique

URL : https://journals.openedition.org/ere/7370

DOI : $10.4000 /$ ere. 7370

ISSN : 2561-2271

Éditeur

Centr'ERE

Référence électronique

Isabel Orellana, «La communauté d'apprentissage en éducation relative à l'environnement : une nouvelle stratégie dans un processus de changements éducationnels », Éducation relative à l'environnement [En ligne], Volume 1 | 1999, mis en ligne le 15 septembre 1999, consulté le 28 mai 2021. URL : http://journals.openedition.org/ere/7370 ; DOI : https://doi.org/10.4000/ere.7370 


\title{
La communauté d'apprentissage en éducation relative à
}

\section{l'environnement : une nouvelle stratégie dans un processus de changements éducationnels}

\author{
Isabel Orellana
}

1 Dans le cadre des changements complexes qui caractérisent la société contemporaine, une nouvelle réflexion s'amorce visant à revaloriser le rôle de l'éducation en tant que facteur clé de transformation sociale (Faure, 1972 ; Morin et Kern, 1993 ; Delors, 1996 ; Fondation pour le Progrès de l'Homme, 1997). La crise universelle de "l'ère planétaire " (Morin et Kern, 1993) atteint tous les aspects de la vie et se manifeste par des problèmes socio-environnementaux de plus en plus cruciaux. L'impact des activités humaines sur le milieu laisse des traces trop souvent néfastes, certains impacts étant irréversibles. Malgré les importants avancements de la science et de la technologie (vus souvent comme la solution aux problèmes), le monde souffre d'un type de développement incapable d'assurer une qualité de vie adéquate où l'être humain puisse s'épanouir et vivre en harmonie avec le milieu de vie.

2 Dans ce contexte, l'éducation relative à l'environnement (ERE), reconnue comme une dimension essentielle de l'éducation fondamentale, joue un rôle clé pour la construction de nouvelles attitudes, conduites et valeurs des personnes et des communautés à l'égard de l'environnement (Unesco-PNUE, 1976, 1978 ; Greenall Gough, 1993 ; Sauvé, 1997). Une véritable responsabilisation individuelle et collective s'impose. À cet égard, la création des communautés d'apprentissage en ERE apparaît comme une stratégie éducative prometteuse. Il s'agit de groupes de travail, formés à l'initiative des institutions éducatives en association avec les membres de la communauté qui se penchent sur un projet commun et entreprennent un processus participatif d'apprentissage et de recherche de solutions aux problèmes environnementaux ou 
socio-environnementaux du milieu de vie (Stapp et coll., 1988). Les communautés d'apprentissage, envisagées comme une stratégie de formation intégrale des personnes et des collectivités, sont de nature à développer un sentiment d'appartenance et l'engagement des personnes et de la communauté envers leur milieu de vie.

En 1992, lors du Forum Global tenu parallèlement au Sommet de la Terre à Rio (Conseil de la Terre, 1993), s'exprime officiellement la nécessité de développer un regard nouveau face aux enjeux environnementaux et sociaux actuels, et leur lien indissociable. Lorsqu'on suit la trajectoire et l'évolution de l'ERE, on constate que celleci émerge après pas moins de trois siècles de domination d'une vision rationaliste, utilitariste et réductionniste à travers laquelle la réalité a été perçue comme une multitude de phénomènes et d'événements séparés, fragmentés. Son évolution marche de pair avec une nouvelle vision du monde qui met en évidence l'imbrication et l'interdépendance des composantes du système-personne - société - nature et qui situe l'être humain au cœur d'interrelations profondes et complexes des éléments politiques, économiques, sociaux, culturels, spirituels, et biophysiques de l'environnement (Orellana et Fauteux, 1998).

Cependant, à travers sa trajectoire historique, l'ERE a été envisagée selon différentes perceptions: comme contenu, comme processus, comme orientation curriculaire, comme discipline, comme approche holistique (Greenall Gough, 1993). Pour certains, elle est caractérisée par une vision instrumentaliste, centrée principalement sur la résolution des problèmes, faisant la promotion de l'écocivisme et de la gestion des problèmes environnementaux pour une utilisation plus rationnelle des ressources naturelles, pour leur protection et leur conservation en vue de maintenir leur potentiel d'exploitation. Pour d'autres, l'ERE est abordée selon une approche intégrale, systémique et holistique, interdisciplinaire, menant à la construction d'un nouveau type de relation avec l'environnement, stimulant l'émergence d'une nouvelle sensibilité à l'égard du milieu de vie, développant des nouvelles capacités d'analyse critique de la réalité, favorisant un agir individuel et collectif plus responsable et respectueux envers l'environnement. Ainsi, la conception d'une nouvelle réalité et la recherche de solutions aux problèmes, sont perçues comme un processus qui requiert un regard critique et une mise en question, au niveau individuel et collectif, de ce qui apparaît comme "certain» de manière à déclencher la créativité et développer la pensée complexe vers le changement.

5 L'éducation, signalait Delors (1996, p. 7), «constitue l'une de plus puissantes armes dont nous disposons pour modeler l'avenir ». Elle est déterminante pour contribuer à une meilleure prise de conscience de la réalité, pour développer une meilleure faculté de discernement à l'égard de celle-ci, pour développer des habiletés à résoudre des problèmes et pour concevoir une nouvelle réalité. L'éducation, développant la capacité de réflexion critique qui permettra une nouvelle lecture de la réalité dans toute sa complexité, ouvrira les voies pour repenser l'agir individuel et collectif de l'être humain. L'acquisition de connaissances n'est plus par conséquent conçue comme un processus de transmission mais comme issue d'un processus complexe de construction de savoirs où interagissent les connaissances individuelles et collectives, quotidiennes et scientifiques (Le Boterf, 1981 ; Morin et Kern, 1993) dans un cadre social et historique en évolution. L'éducation ne peut donc pas être envisagée comme étant du ressort exclusif de l'école et du système scolaire. 
6 S'inscrivant dans cette vision et suivant une orientation de critique sociale, l'éducation relative à l'environnement propose de nouvelles approches pédagogiques; celles-ci visent la responsabilisation et la participation active, consciente et concertée, de toutes les composantes de la société vers une nouvelle relation avec l'environnement par l'engagement dans un processus de construction d'une nouvelle réalité sociale et environnementale. Ainsi, le concept de société éducative, déjà proposé par Faure en 1972 dans son rapport de la Commission internationale sur l'éducation, reprend toute sa pertinence. Ce concept fait appel à l'implication planifiée de la société toute entière dans un processus éducatif global. Ceci implique la participation active de tous les intervenants de la vie sociale, l'état et ses institutions, l'entreprise, les organismes communautaires, les individus. Dans ce contexte, l'intégration active de l'école dans la société et le recours à ses différentes composantes et à tous les acteurs sociaux dans une démarche éducative intégratrice, crée des conditions propices au développement d'un processus de changement où chaque personne se forme comme acteur responsable et actif, où l'école se transforme en une école communautaire et responsable (Bertrand et Valois, 1992) en constante évolution. Le processus d'apprentissage devient un processus de prise de conscience, de responsabilisation, où se forgent les transformations, les changements sociaux (Freire, 1974).

7 La société éducative représente un véritable défi social, qui vise à transformer le processus éducatif en processus permanent où chaque personne et organisme participe activement à l'éducation et à la formation, tout au long de leur vie (Antikainen et coll., 1996). Par ce processus, on envisage une construction continue de la personne, de son savoir et de ses attitudes, ainsi que de sa faculté d'engagement et d'action. D'après Faure (1972), la société éducative constitue une stratégie visant à engager la société entière dans un processus éducatif. Elle repose sur l'idée d'une osmose entre l'éducation et la société où " l'apprentissage est l'affaire de toute une vie à la fois dans sa durée et dans sa diversité»; c'est un apprentissage permanent. Toutes les composantes de la société sont appelées à jouer un rôle éducatif et à contribuer à la construction d'une « société éducative consciente » (Deleon, 1996). L'éducation est ainsi perçue comme un processus inhérent à un système où s'exerce la démocratie participative (Ranson, 1994). Ce processus se concrétise dans les différents secteurs de la société par la création des communautés éducatives.

8 Les communautés éducatives se forment par le développement des liens d'échange et de collaboration entre organismes éducatifs et membres de la communauté (familles, entreprises, $\mathrm{ONG}, \mathrm{OG}$,) autour des problématiques communes existantes dans un milieu de vie et pour l'amélioration de sa qualité. Buttner (1995) présente ce lien actif comme un processus de construction de connaissances dans la perspective d'une nouvelle éthique, celle d'une civilisation écologique. Une telle stratégie est privilégiée par l'ERE (Torres, 1996 ; Sauvé, 1997).

9 L'éducation relative à l'environnement place l'école (ou autre instance éducative) au centre d'une dynamique communautaire en vue d'un développement intégral des élèves - étudiants et d'un développement optimal de la communauté. Dans ce même sens, l'ERE propose la formation d'unités fonctionnelles au sein de ces communautés, soit de groupes de travail qui associent le milieu éducatif et la communauté et s'organisent autour d'un projet concret, bâtissant un processus d'apprentissage en contexte interdisciplinaire et en complémentarité de savoirs : ce sont les communautés d'apprentissage, articulées autour d'un problème du milieu de vie, à la recherche de 
solutions, ou autour d'un projet visant le développement d'un aspect particulier de la vie communautaire. S'appuyant sur des approches expérientielle, holistique, systémique, interdisciplinaire et coopérative, se met en marche un processus de coapprentissage (associé à une investigation commune) qui favorise l'émergence d'un véritable processus de changement individuel et collectif. La figure 1 illustre l'organisation du réseau de notions associées aux communautés d'apprentissage.

\section{Figure 1 : Organisation du réseau de notions associées aux communautés d'apprentissage}

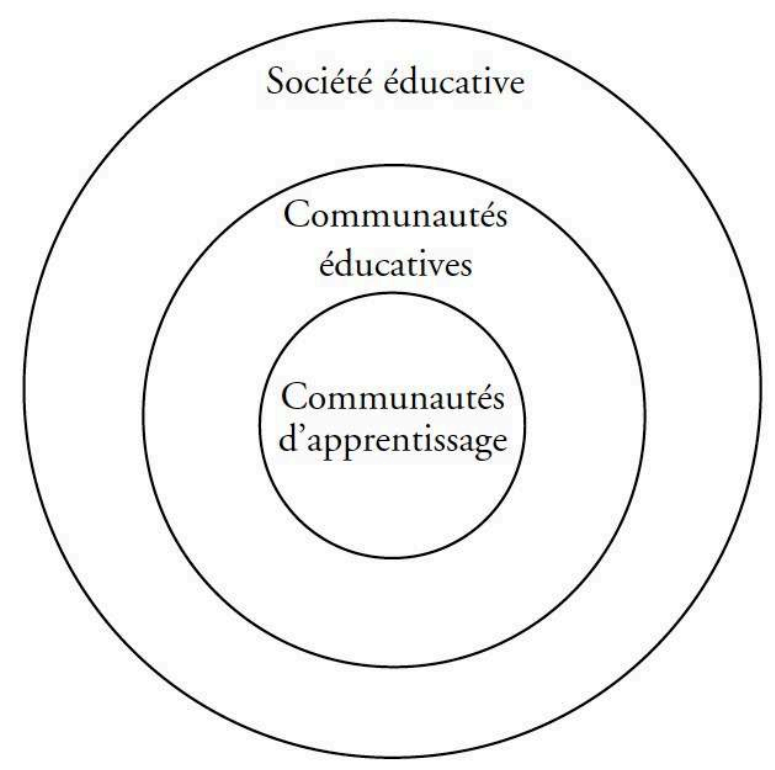

Par la participation collaborative, active et critique de membres des institutions éducatives et de la communauté, associés dans une communauté d'apprentissage, il se produit un enrichissement complémentaire entre le savoir préalable, l'expérience perceptive et existentielle du collectif, la créativité collective, et le partage des ressources et des moyens. Il s'agit d'un processus de changement planifié qui fait appel aux efforts conjoints, conscients et délibérés pour produire la transformation souhaitée (Savoie-Zajc, 1993). Mais, il s'agit également d'un processus de changement émergent par l'effet du dialogue, de la complémentarité, de la mise en commun des connaissances quotidiennes et scientifiques. Ces changements (planifiés et émergents) se manifestent dans le milieu éducatif, le milieu de vie social et environnemental et chez les personnes dont le développement des aspects cognitifs, affectifs, sociaux et moraux aura été favorisé (Sauvé, 1997).

Plusieurs auteurs (Spies-Bong, 1989 ; Gabelnick, 1990 ; McCaleb, 1994 ; Potapchuk et Polk, 1994 ; Wells et Fullan, 1994 ; Buttner, 1995 ; Antikainen et coll., 1996) ont abordé la formation des communautés d'apprentissage comme une approche stratégique éducative prometteuse face à l'ampleur des problèmes socio-environnementaux et aux importants changements institutionnels de décentralisation et d'autonomie. Par la dynamique du dialogue des savoirs et de co-formation collaborative, associée à une implication active dans le milieu de vie, cette stratégie favorise le développement du sentiment d'appartenance et d'identité et l'engagement dans un processus de 
changement pour une meilleure qualité de vie. Il se crée alors des conditions propices à la formation des personnes responsables de leur devenir.

Cependant, les expériences de mise en application des communautés d'apprentissage sont relativement récentes. Peu d'études se sont attardées à l'analyse de leurs théories et de leurs pratiques. Les constats des expériences d'application de celle-ci témoignent d'une diversité d'appellations (Productions Tact, 1996 ; Grégoire, 1997) : communauté de collaboration, unité éducative, communauté d'apprentissage, communauté d'investigation, communauté de recherche. On retrouve aussi les termes anglais, communit of learners, learning community, community of inquiry, community of practice, etc. lesquelles semblent présenter des caractéristiques communes: fondements épistémologiques, structure et organisation, approches pédagogiques, stratégies de travail et mise en application. Chacune de ces appellations a cependant des spécificités ; certaines évoquent davantage la dimension structurelle et organisationnelle, et d'autres, plus en lien avec le concept d'apprentissage, renvoient aux processus de coconstruction de connaissances; d'autres enfin, axées sur le concept de communauté, mettent l'accent sur le processus collectif de travail, de coparticipation, de collaboration, de coopération (Grégoire, 1997). Il reste cependant que les traits communs et les similitudes, les convergences des fondements, laissent croire à l'émergence d'un seul nouveau concept en voie de construction, d'une seule nouvelle stratégie éducative. S'ouvre ici tout un champ de recherche visant les conceptions et pratiques relatives à ce que nous appellerons les « communautés d'apprentissage ».

\section{BIBLIOGRAPHIE}

Antikainen, A., Houtsonen, J., Huotelin, H. et Kauppila, J. (1996). Living in a learning society : Lifestories, identities and education. London et Washington : The Falmer Press.

Bertrand Y. et Valois, P. (1992). École et sociétés. Québec : Éd. Agence d'Arc.

Buttner, P. (1995). Necessidade e elementos de um novo paradigma em prol da educação holística. Revista de Educação pública, 4(5), 236-248.

Conseil de la Terre. (1993). Traité : L'éducation environnementale pour des sociétés durables et une responsabilité globale. Rio de Janeiro, Brésil : Conseil de la Terre.

Deleon, A. (1996). Un quart de siècle après. Le Courrier de l'UNESCO, avril, 12-16.

Delors, J. (1996). Former les acteurs du futur. Le Courrier de l'UNESCO, avril, 6-11. Faure, E. (1972). Apprendre à être ? Rapport de la Commission internationale sur le développement de l'éducation. Paris : A. Fayard et Unesco.

Fondation pour le Progrès de l'Homme. (1997). Alliance pour un monde responsable et solidaire. Paris, France : Fondation pour le Progrès de l'Homme.

Freire, P. (1974). La educación como práctica de la libertad. México : Ed. Siglo Veintiuno.

Gabelnick, F. (1990). Learning communities : creating connections among students, faculty and disciplines. New directions for teaching and learning. San Francisco : Jossey-Bass. 
Greenall Gough, A. (1993). Founders in environmental education. Australia : Deakin Uni-versity Press.

Grégoire, R. (1997). Communauté d'apprentissage. Attitudes fondamentales. Université Laval : Productions Tact, site internet Tact.

Le Boterf, G. (1981). L'enquête participation en question : analyse d'une expérience, description d'une méthode et réflexions critiques. Paris : Ligue française de l'enseignement et de l'éducation permanente.

McCaleb, S.P. (1994). Building communities of learners. A collaboration among teachers, stu-dents, families and community. New College of California et New York : St. Martin's Press.

Morin, E. et Kern, A.B. (1993). Terre-Patrie. France : Éditions du Seuil.

Orellana, I. et Fauteux, S. (1998). L'éducation relative à l'environnement à travers les grands moments de son histoire. Proceedings from the On-line Colloquium : The Future of Environmental Education in a Postmodern World ? 19 october 1998.

Potapchuk, R.W. et Polk, C.G. (1994). Building collaborative community. Washington : National Institute for Dispute Resolution.

Productions Tact. (1996). Communauté d'apprentissage : des mots pour dire la chose. Compterendu de l'atelier Apprendre au 21e siècle : 19 - 21 avril 1996, King City.

Ranson, S. (1994). Towards the learning society. London : Cassell.

Sauvé, L. (1997, 2éd.). Pour une éducation relative à l'environnement. Montréal : Éd. Guérin.

Savoie-Zajc, L. (1993). Les modèles de changement planifié en éducation. Québec : Les Éditions Logiques.

Spies-Bong, G. (1989). Pour une pédagogie en communauté de vie. Le petit plan d'Iéna de Peter Petersen. Paris et Bruxelles : Éd. Universitaires et De Boeck-Wesmael.

Stapp, W., Bull, J. et coll. (1988). Education in action. A community problem solving program for schools. Michigan : Ed Thompson-Shore Inc.

Torres, M. (1996). La dimensión ambiental : un reto para la educación de la nueva sociedad. Proyectos ambientales escolares. Santafé de Bogotá : Ministerio de la Educación Nacional de Colombia.

Unesco-PNUE. (1978). Rapport final, Conférence intergouvernementale sur l'éducation relative à l'environnement, Tbilissi (URSS), 14-26 oct. 1977. Paris : Unesco-PNUE.

Unesco-PNUE. (1976) La Chartre de Belgrade. Connexion, 1 (1).

Wells, G. et Fullan, M. (1994). Changing school from within creating communities of inquiry. Toronto, Canada : The Ontario Institute for Studies in Education.

\section{AUTEUR}

\section{ISABEL ORELLANA}

Isabel Orellana est étudiante au Doctorat en éducation (spécialisation en éducation relative à l'environnement) à l'UQAM et titulaire d'une maîtrise en sciences de l'environnement. Membre de CIRADE, elle œuvre dans le domaine de l'éducation relative à l'environnement depuis environ 10 ans, auprès d'ONG et d'institutions d'éducation, dans des projets nationaux et internationaux. 